\title{
Studies in radical theory for restricted classes of associative rings
}

\author{
N.R. MCCONNELL
}

This thesis is a study of radical ideals in restricted domains of associative rings.

The first chapter introduces a generalisation of the concept of strictness, and studies, for a given radical class, the class of rings for which the radical is hereditary and/or strict (in the general sense). This leads to some results on embedding radical classes in generalised strict radical classes, and on degrees of hereditariness of radical classes.

In the second chapter, the ideals of a ring which can be radical ideals are studied, with characterisations being given for Dedekind domains and some of their extension rings. This characterises radical ideals for a fairly wide class of integral domains, and thus goes some way toward characterising supernilpotent radical ideals of commutative rings.

In the third chapter, a study is made of radical classes of commutative rings, with a characterisation being given of the strongly hereditary strict radicals, and the implications of restricting to commutativity are considered for some other results and problems. There is also an investigation of the permanent radical - that is, the part of the radical of a ring which remains as part of the radical of any extension - for more general classes of rings.

The thesis thus gives an insight into the behaviour of radical classes within single rings rather than globally, which gives us more information about the nature of radical classes.

Department of Mathematics and Computing

University College of Central Queensland

Rockhampton MC QLD 4702

Australia

Received 4th March 1991

Thesis submitted to The University of Tasmania, May 1990. Degree approved November 1990. Supervisor: Dr B.J. Gardner.

Copyright Clearance Centre, Inc. Serial-fee code: 0004-9729/91 \$A2.00+0.00. 